\title{
3D Gas Dynamics from Methanol Masers observed with the EVN reveals Rotating Disks around O-type Young Stars
}

\author{
Ciriaco Goddi* \\ Joint Institute for VLBI in Europe, Postbus 2, NL-7990 AA Dwingeloo, the Netherlands \\ E-mail: cgoddiejive.nl
}

Luca Moscadelli

INAF-Osservatorio Astrofisico di Arcetri, Largo E. Fermi 5, 50125 Firenze, Italy

E-mail: moscaearcetri.astro.it

\begin{abstract}
NGC7538 IRS1 is considered the best high-mass accretion disk candidate around an O-type young star in the northern hemisphere. We investigated the 3D kinematics and dynamics of circumstellar gas with very high linear resolution, from tens to $1500 \mathrm{AU}$, with the ultimate goal of building a comprehensive dynamical model for this YSO. We employed four different observing epochs of EVN data at $6.7 \mathrm{GHz}$, spanning almost eight years, which enabled us to measure, besides line-of-sight (1.o.s.) velocities and positions, also l.o.s. accelerations and proper motions of methanol masers. In addition, we imaged with the JVLA-B array highly-excited ammonia inversion lines, from $(\mathrm{J}, \mathrm{K})=(6,6)$ to $(14,14)$, which enabled us to probe the hottest molecular gas very close to the exciting source(s). We found five $6.7 \mathrm{GHz}$ maser clusters which are distributed over a region extended N-S across $\sim 1500 \mathrm{AU}$ and are associated with three peaks of the radio continuum. We proposed that these maser clusters identify three individual high-mass YSOs, named IRS1a, IRS1b, and IRS1c. We modeled the maser clusters in IRS1a and IRS1b in terms of edge-on disks in centrifugal equilibrium, demonstrating quasi-Keplerian rotation around IRS1a, a high-mass YSO of up to $25 \mathrm{M}_{\odot}$. In summary, we present compelling evidence that NGC7538 IRS1 is not forming just one single high-mass YSO, but consists of a multiple system of highmass YSOs, which are surrounded by accretion disks.
\end{abstract}

12th European VLBI Network Symposium and Users Meeting,

7-10 October 2014

Cagliari, Italy

\footnotetext{
* Speaker.
} 


\section{Introduction}

Despite the prominent role played by high-mass stars in the evolution of galaxies, there is no general theory of high-mass star formation (HMSF). The main difficulty arises because the intense radiation pressure from the stellar luminosity and the thermal pressure from the HII region around the massive young stellar objects (MYSOs) may be sufficient to prevent matter from reaching the star. Recent theoretical studies have however demonstrated that if accretion occurs through a circumstellar disk, stars up to $140 M_{\odot}$ can still form [1]. Despite the theoretical evidence, high extinction, clustering, and large distances of high-mass star forming regions (HMSFRs) hinder attempts to resolve the circumstellar gas at small radii $(<1000 \mathrm{AU})$ where outflows are believed to be launched and collimated from accretion disks.

In this context, one excellent diagnostic tool of gas kinematics within 10-1000 AU from MYSOs is provided by multi-epoch very long baseline interferometric (VLBI) observations of interstellar masers [2, 3, 4, 5, 6, 7, 8]. While $\mathrm{SiO}$ masers are rarely found in HMSFRs $[9,10]$, the $\mathrm{H}_{2} \mathrm{O}$ masers are ubiquitous and generally trace shocked gas in both low-mass and high-mass protostellar outflows [11, 12]. Among different molecular masers, $\mathrm{CH}_{3} \mathrm{OH}$ is particularly interesting, because it is exclusively associated with HMSF and provides an excellent probe of accretion [13]. Recently, [14] reported a direct measurement of infall of a circumstellar envelope with a radius of only $300 \mathrm{AU}$ around an intermediate-mass protostar by using multi-epoch VLBI observations of $\mathrm{CH}_{3} \mathrm{OH}$ masers spanning six years. However, in order to constrain theoretical models of HMSF, observational signatures of mass accretion in more massive O-type forming stars are desirable.

\section{NGC7538 IRS1}

At a distance of $2.7 \mathrm{kpc}$ [15], NGC 7538 IRS1 has a luminosity equivalent to an O6/7 ZAMS star $\left(8 \times 10^{4} L_{\odot}\right)$. Recently, a number of interferometric studies conducted at $3.4 \mathrm{~mm}, 1.3 \mathrm{~mm}$ and at $0.8 \mathrm{~mm}$ with CARMA, PdBI, and SMA ( $3^{\prime \prime}-0.2$ angular resolutions), detected typical hot-core species, showing inverse P-Cygni profiles, probing inward gas motion with $\dot{M} \sim 10^{-3} M_{\odot} \mathrm{yr}^{-1}$ on scales $\gtrsim 1000 \mathrm{AU}([16,17,18])$. Radio and mm studies also identified several outflows, along N-S [19], NW-SE [16], and NE-SW [18], respectively. The simultaneous presence of a jet/outflow and strong accretion flow toward IRS 1, led some authors to postulate the presence of an accretion disk, whose evidence is mainly based on VLBI imaging of methanol masers [20, 21, 22]. Competing models have been however proposed by different groups, to explain positions and l.o.s. velocities of $\mathrm{CH}_{3} \mathrm{OH}$ maser spots in the region, involving disk/outflow systems with different orientations, which led to some controversy in the interpretation of this region.

\section{Results}

By combining multi-epoch observations of $6.7 \mathrm{GHz} \mathrm{CH}_{3} \mathrm{OH}$ masers with the $\mathrm{EVN}$ and highresolution imaging of $\mathrm{NH}_{3}$ inversion lines with the JVLA, we investigated the 3D kinematics and physical conditions of circumstellar gas with linear resolutions from tens to $1500 \mathrm{AU}$, with the ultimate goal of building a comprehensive dynamical model for NGC 7538 IRS1, considered the best high-mass accretion disk candidate around an O-type young star in the northern hemisphere. 

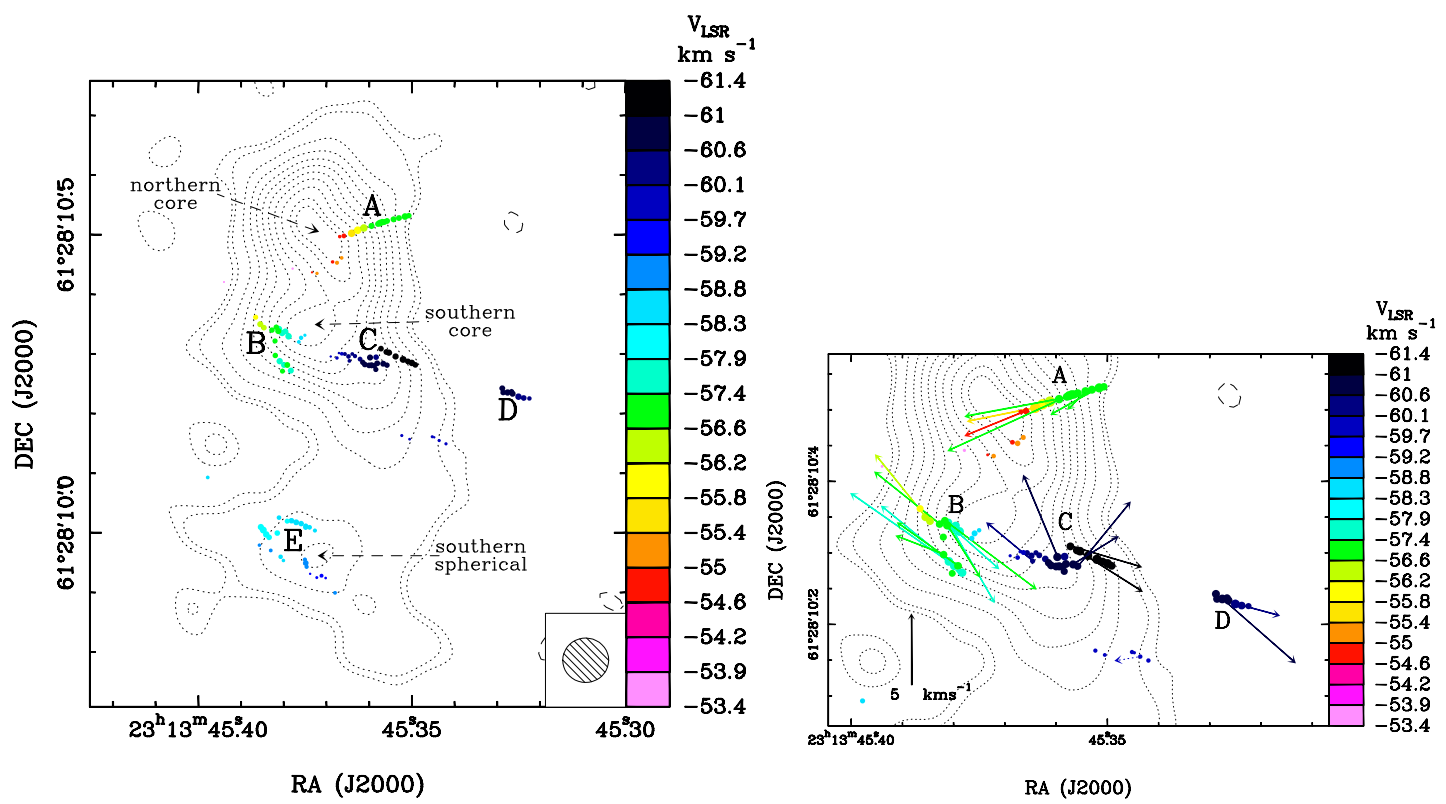

Figure 1: $6.7 \mathrm{GHz} \mathrm{CH}_{3} \mathrm{OH}$ masers detected over three epochs with the EVN, overlaid on the $1.3 \mathrm{~cm}$ continuum imaged with the VLA A-Array. Colored dots show the absolute position of individual maser features, with colors denoting their $V_{\text {LSR }}$. Masers are grouped in different clusters, labeled using capital letters from "A" to "E". The $1.3 \mathrm{~cm}$ continuum (Dotted contours) was imaged with the VLA-A array (originally reported

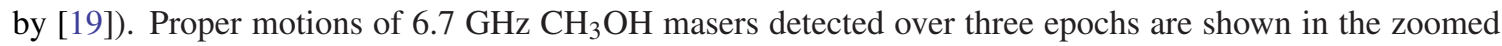
view in the right panel with colored arrows (dotted arrows denote the most uncertain measurements).

\subsection{EVN observations of 6.7 $\mathrm{GHz} \mathrm{CH}_{3} \mathrm{OH}$ masers over 2002-2009}

We reduced and analyzed four individual $\mathrm{EVN}$ datasets of $6.7 \mathrm{GHz} \mathrm{CH}_{3} \mathrm{OH}$ masers observed at four distinct observing epochs over the years 2002-2009. A full report on the results is presented in [23]. Figure 1 shows the spatial and $V_{\mathrm{LSR}}$ distribution of the $6.7 \mathrm{GHz} \mathrm{CH} \mathrm{CH}_{3} \mathrm{OH}$ masers in $\mathrm{NGC7538}$ IRS1, overlaid on a map of the $1.3 \mathrm{~cm}$ continuum emission imaged with the VLA A-Array. Spread over an area of $\Delta \alpha \times \Delta \delta \approx 0$ "' $4 \times 0$ "' 6 , most of the $6.7 \mathrm{GHz}$ maser features are organized in five distinct clusters, which are labelled with capital letters from "A" to "E" in Figure 1. There is clearly a good positional correspondence between the maser clusters and three components of the radio continuum emission. We propose that these maser clusters identify three individual high-mass YSOs in NGC7538 IRS1, named IRS1a (associated with clusters "B" and "C"), IRS1b (associated with cluster "A"), and IRS1c (associated with cluster "E"). We find that the $6.7 \mathrm{GHz}$ masers distribute along a line with a regular variation of $V_{\mathrm{LSR}}$ with position along the major axis of the distribution of maser cluster "A" and the combined clusters "B"+ " $\mathrm{C}$ ". We measure proper motions for 33 maser features, which have an average amplitude $\left(4.8 \pm 0.6 \mathrm{~km} \mathrm{~s}^{-1}\right)$ similar to the variation in $V_{\mathrm{LSR}}$ across the maser cluster, and are approximately parallel to the clusters' elongation axes (see right panel of Fig. 1). By studying the time variation of the maser spectrum, we derive also l.o.s. accelerations for 30 features, with typical amplitude of $\sim 10^{-3}-10^{-2} \mathrm{~km} \mathrm{~s}^{-1} \mathrm{yr}^{-1}$. We model the masers in both clusters "A" and "B"+" $\mathrm{C}$ " in terms of an edge-on disk in centrifugal equilibrium (see section 5 in [23] for more details). Based on our modeling, masers of clusters "B"+ "C" may trace a quasi-Keplerian $\sim 1 M_{\odot}$, thin disk, orbiting around a high-mass YSO, IRS1a, of up to $\approx 25 M_{\odot}$. 
The disk traced by the masers of cluster " $\mathrm{A}$ " is both massive ( $\lesssim 16 M_{\odot}$, inside a radius of $\left.\approx 500 \mathrm{AU}\right)$ and thick (opening angle $\approx 45^{\circ}$ ), and the mass of the central YSO, IRS1b, is constrained to be at most a few $M_{\odot}$. Towards cluster "E", $\mathrm{NH}_{3}$ and $6.7 \mathrm{GHz}$ masers trace more quiescent dynamics than for the other clusters. The presence of a radio continuum peak suggests that the YSO associated with the cluster "E", IRS1c, may be an ionizing, massive YSO as well.

\subsection{JVLA imaging of $\mathrm{NH}_{3}(6,6)$ to $(14,14)$}

Using the JVLA in B configuration, we imaged at a resolution of 0. " $15-0$ ". 3 seven $\mathrm{NH}_{3}$ lines with energy levels high above the ground state (equivalent to $400-1950 \mathrm{~K})$, from $(J, K)=(6,6)$ to $(14,14)$, in NGC7538 IRS1. A complete analysis of the $\mathrm{NH}_{3}$ data is presented in [24]. The highlyexcited $\mathrm{NH}_{3}$ inversion transitions are observed in absorption against the strong HC-HII region associated with NGC7538 IRS1. Figure 2 shows an overlay of $\mathrm{NH}_{3}$ absorption peak positions in individual spectral channels (fitted with Gaussians) for four different inversion lines and $\mathrm{CH}_{3} \mathrm{OH}$ maser spots onto the $1.3 \mathrm{~cm}$ radio continuum imaged with the VLA at 0 "'08 resolution. The $\mathrm{NH}_{3}$ absorption peaks clearly identify a velocity gradient close to N-S, i.e. the direction along the line connecting the two components of the radio continuum separated by $500 \mathrm{AU}$ or 0.2 , the northern and southern core components. This reflects the global rotation of the natal massive core, in the orbital plane of the binary composed by the two high-mass YSOs IRS1a and IRS1b. With an extent of $\sim 15 \mathrm{~km} \mathrm{~s}^{-1}$, this velocity gradient corresponds to an enclosed mass in the core of $\sim 42 M_{\odot}$ (assuming the $\mathrm{NH}_{3}$ gas is rotating in centrifugal equilibrium), in good agreement with the total dynamical mass estimated from VLBI measurements of $\mathrm{CH}_{3} \mathrm{OH}$ masers $\left(41 M_{\odot}\right)$.

From simple LTE analysis, the molecular gas in the core has a temperature of $280 \mathrm{~K}$, with a potential hotter component up to $500 \mathrm{~K}$. The $\mathrm{H}_{2}$ density is over $10^{10} \mathrm{~cm}^{-3}$ (assuming $\left[\mathrm{NH}_{3}\right] /\left[\mathrm{H}_{2}\right]=10^{-7}$ ), making NGC7538 IRS1 the densest hot core known. We also estimate a gas mass from $\mathrm{NH}_{3}$ of 19 $34 M_{\odot}$ for the core, which is lower than the "total" (stars + gas) dynamical masses estimated from $\mathrm{NH}_{3}$ and $\mathrm{CH}_{3} \mathrm{OH}$ masers. The southern component of the continuum (located by the "E" cluster) has a lower temperature, around hundred K, but similar (though lower) molecular gas density.

\section{Conclusions}

This study on NGC7538 IRS1, demonstrates that the combination of multi-epoch VLBI measurements of $\mathrm{CH}_{3} \mathrm{OH}$ masers and high-angular (i.e. a few tenths of arcseconds) resolution imaging of high-excitation lines of $\mathrm{NH}_{3}$ at $\sim 1 \mathrm{~cm}$ wavelengths is a powerful tool to study the kinematics and physical conditions of the hottest and densest molecular gas in the vicinity of the central high-mass $\mathrm{YSO}(\mathrm{s})$, that is in accretion disks and the innermost regions of circumstellar envelopes.

In the specific case of NGC7538 IRS1, we find compelling evidence that this object is not forming just one single MYSO, but consists of a multiple system of MYSOs, which are surrounded by accretion disks, and are probably driving individual outflows. This new model naturally explains all the different orientations and disk/outflow structures proposed for the region in previous models.

\section{References}

[1] Kuiper, R., Klahr, H., Beuther, H., \& Henning, T. 2011, Circumventing the Radiation Pressure Barrier in the Formation of Massive Stars via Disk Accretion, ApJ, 732, 20 


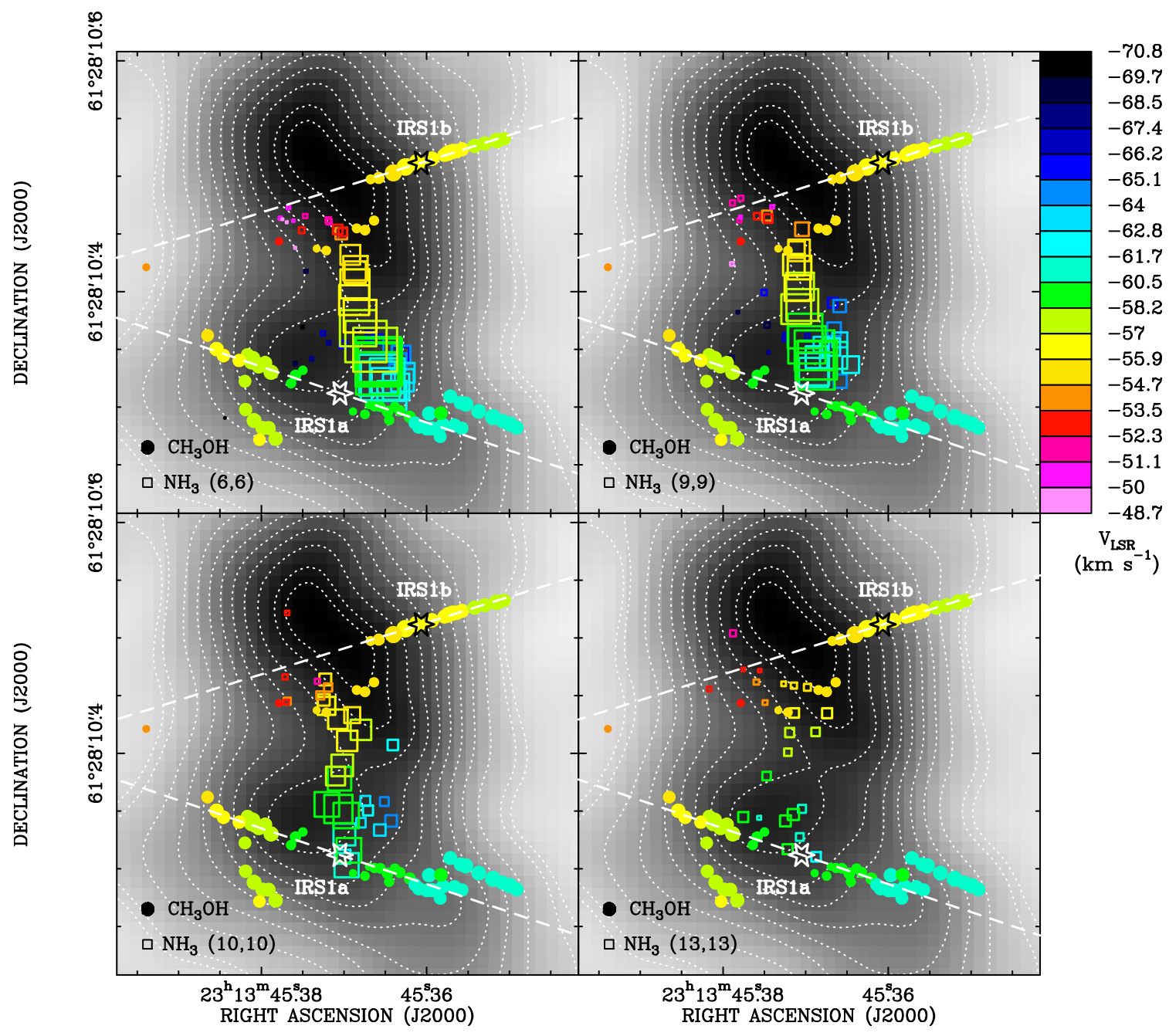

Figure 2: Fitted emission centroids of $\mathrm{NH}_{3}$ (open squares) and $\mathrm{CH}_{3} \mathrm{OH}$ masers (filled circles) overlaid on the the $1.3 \mathrm{~cm}$ continuum map (black image and white contours). Color denotes $V_{\mathrm{LSR}}$ (color scale on the right-hand side). The sizes of squares and circles scale linearly and logarithmically with the flux density of $\mathrm{NH}_{3}$ absorption and $\mathrm{CH}_{3} \mathrm{OH}$ maser emission, respectively. The relative alignment between $\mathrm{NH}_{3}$ and $\mathrm{CH}_{3} \mathrm{OH}$ is $\mathrm{s}$ accurate to $\sim 30$ mas. Linear fits to the spatial distributions of maser features in cluster " $\mathrm{A}$ ", and in the combined clusters "B"+"C" (dashed lines) are indicated by dashed white lines. The stars labeled IRS1a and IRS1b mark the YSO positions, as discussed in the text. Note that $\mathrm{NH}_{3}$ absorption distributes between IRS1a and IRS1b, shows a velocity gradients roughly N-S, and is strongest towards IRS1a.

[2] Goddi, C., \& Moscadelli, L. 2006, Tracing the base of protostellar wind(s) towards the high-mass star forming region AFGL 5142, A\&A, 447, 577

[3] Goddi, C., Moscadelli, L., Torrelles, J. M., Uscanga, L., \& Cesaroni, R. 2006, Evidence supporting the kinematic interpretation of water maser proper motions, A\&A, 447, L9

[4] Goddi, C., Moscadelli, L., Sanna, A., Cesaroni, R., \& Minier, V. 2007, Associations of $\mathrm{H}_{2} \mathrm{O}$ and $\mathrm{CH}_{3} \mathrm{OH}$ masers at milli-arcsec angular resolution in two high-mass YSOs, A\&A, 461, 1027

[5] Matthews, L. D., Greenhill, L. J., Goddi, C., et al. 2010, A Feature Movie of SiO Emission 20-100 AU from the Massive Young Stellar Object Orion Source I, ApJ, 708, 80 
[6] Moscadelli, L., Goddi, C., Cesaroni, R., Beltrán, M. T., \& Furuya, R. S. 2007, Massive star-formation in G24.78+0.08 explored through VLBI maser observations, A\&A, 472, 867

[7] Sanna, A., Moscadelli, L., Cesaroni, R., et al. 2010, VLBI study of maser kinematics in high-mass star-forming regions. I. G16.59-0.05, A\&A, 517, AA71

[8] Sanna, A., Moscadelli, L., Cesaroni, R., et al. 2010, VLBI study of maser kinematics in high-mass star-forming regions. II. G23.01-0.41, A\&A, 517, AA78

[9] Goddi, C., Greenhill, L. J., Humphreys, E. M. L., et al. 2009, A 42.3-43.6 GHz Spectral Survey of Orion $B N / K L, A p J, 691,1254$

[10] Goddi, C., Greenhill, L. J., Chandler, C. J., et al. 2009, Maser Emission from SiO Isotopologues Traces the Innermost 100 AU Around Radio Source I in Orion Becklin-Neugebauer/Kleinmann-Low, ApJ, 698, 1165

[11] Moscadelli, L., Testi, L., Furuya, R. S., et al. 2006, First results from a VLBA proper motion survey of $\mathrm{H}_{2} \mathrm{O}$ masers in low-mass YSOs: the Serpens core and $\mathrm{RNO}$ 15-FIR, A\&A, 446, 985

[12] Goddi, C., Moscadelli, L., Alef, W., et al. 2005, Kinematics of $\mathrm{H}_{2} \mathrm{O}$ masers in high-mass star forming regions, $A \& A, 432,161$

[13] Moscadelli, L., Sanna, A., \& Goddi, C. 2011, Unveiling the gas kinematics at 10 AU scales in high-mass star-forming regions., A\&A, 536, AA38

[14] Goddi, C., Moscadelli, L., \& Sanna, A. 2011, Infall and outflow within 400 AU from a high-mass protostar. 3D velocity fields from methanol and water masers in AFLG 5142, A\&A, 535, LL8

[15] Moscadelli, L., Reid, M. J., Menten, K. M., et al. 2009, Trigonometric Parallaxes of Massive Star-Forming Regions. II. Cep A and NGC 7538, ApJ, 693, 406

[16] Qiu, K., Zhang, Q., \& Menten, K. M. 2011, Outflows, Accretion, and Clustered Protostellar Cores Around a Forming $O$ Star, ApJ, 728, 6

[17] Zhu, L., Zhao, J.-H., Wright, M. C. H., et al. 2013, Subarcsecond Observations of NGC 7538 IRS 1: Continuum Distribution and Dynamics of Molecular Gas, ApJ, 779, 51

[18] Beuther, H., Linz, H., \& Henning, T. 2013, Fragmentation, infall, and outflow around the showcase massive protostar NGC 7538 IRS1 at 500 AU resolution, A\&A, 558, AA81

[19] Gaume, R. A., Goss, W. M., Dickel, H. R., Wilson, T. L., \& Johnston, K. J. 1995, The NGC 7538 IRS 1 region of star formation: Observations of the $H 66$ alpha recombination line with a spatial resolution of $300 \mathrm{AU}, \mathrm{ApJ}, 438,776$

[20] Minier, V., Booth, R. S., \& Conway, J. E. 1998, Observations of methanol masers in NGC7538: probable detection of a circumstellar disc, A\&A, 336, L5

[21] Pestalozzi, M. R., Elitzur, M., Conway, J. E., \& Booth, R. S. 2004, A Circumstellar Disk in a High-Mass Star-forming Region, ApJL, 603, L113

[22] Surcis, G., Vlemmings, W. H. T., Torres, R. M., van Langevelde, H. J., \& Hutawarakorn Kramer, B. 2011, A\&A, 533, AA47

[23] Moscadelli, L., \& Goddi, C. 2014, A multiple system of high-mass YSOs surrounded by disks in NGC 7538 IRSI, A\&A, 566, AA150

[24] Goddi, C., Zhang, Q., \& Moscadelli, L. 2015, Hot ammonia around young O-type stars. I. JVLA imaging of $\mathrm{NH}_{3}(6,6)$ to $(14,14)$ in NGC 7538 IRS1, A\&A, 573, AA108 